University of Wollongong

Research Online

Faculty of Engineering and Information

Faculty of Engineering and Information

Sciences - Papers: Part B

Sciences

2017

Three-Dimensional Kinematic Modeling of Helix-Forming Lamina-Emergent Soft Smart Actuators Based on Electroactive Polymers

Rahim Mutlu

University of Wollongong, rmutlu@uow.edu.au

Gursel Alici

University of Wollongong, gursel@uow.edu.au

Weihua Li

University of Wollongong, weihuali@uow.edu.au

Follow this and additional works at: https://ro.uow.edu.au/eispapers1

Part of the Engineering Commons, and the Science and Technology Studies Commons

Research Online is the open access institutional repository for the University of Wollongong. For further information contact the UOW Library: research-pubs@uow.edu.au 


\title{
Three-Dimensional Kinematic Modeling of Helix-Forming Lamina-Emergent Soft Smart Actuators Based on Electroactive Polymers
}

\author{
Abstract \\ Robotic systems consisting of rigid elements connected to each other with single degree of freedom \\ joints have been studied extensively. Robotic systems made of soft and smart materials are expected to \\ provide a high dexterity and adaptability to their physical environment, like their biological counterparts. \\ Electroactive polymer (EAP) actuators, also known as artificial muscles, which can operate both in wet \\ and in dry environments with their promising features such as a low foot-print in activation and energy \\ consumption, suitability to miniaturization, noiseless, and fully compliant operation can be employed to \\ articulate a soft robotic system. This paper reports on kinematic modeling of a polypyrrolebased EAP \\ actuator which is designed and fabricated to form helical configurations in 3-D from its initially spiral 2-D \\ configuration. Denavit-Hartenberg transformations are combined with the backbone model of the \\ actuator to establish the kinematic model. A parametric model has then been incorporated into the \\ kinematic model to accurately estimate the helical configurations of the EAP actuator as a function of \\ time under an electrical input. Experimental and simulation results, which are in good correlation, suggest \\ that the proposed modeling approach is effective enough to estimate the 3-D helical configurations of the \\ EAP actuator.

\section{Disciplines} \\ Engineering | Science and Technology Studies

\section{Publication Details} \\ Mutlu, R., Alici, G. \& Li, W. (2017). Three-Dimensional Kinematic Modeling of Helix-Forming Lamina- \\ Emergent Soft Smart Actuators Based on Electroactive Polymers. IEEE Transactions On Systems, Man, \\ and Cybernetics: Systems, 47 (9), 2562-2573.
}




\title{
3D Kinematic Modelling of Helix Forming Lamina-emergent Soft Smart Actuators Based on Electroactive Polymers
}

\author{
Rahim Mutlu, Gursel Alici and Weihua Li
}

\begin{abstract}
Robotic systems consisting of rigid elements connected to each other with single degree of freedom joints have been studied extensively. Robotic systems made of soft and smart materials are expected to provide a high dexterity and adaptability to their physical environment, like their biological counterparts. Electroactive polymer (EAP) actuators, also known as artificial muscles, which can operate both in wet and dry environments with their promising features such as a low foot-print in activation and energy consumption, suitability to miniaturization, noiseless and fully compliant operation can be employed to articulate a soft robotic system. This paper reports on kinematic modelling of a polypyrrole (PPy) based EAP actuator which is designed and fabricated to form helical configurations in 3D from its initially spiral 2D configuration. Denavit-Hartenberg transformations are combined with the backbone model of the actuator to establish the kinematic model. A parametric model has then been incorporated into the kinematic model to accurately estimate the helical configurations of the EAP actuator as a function of time under an electrical input. Experimental and simulation results, which are in good correlation, suggest that the proposed modeling approach is effective enough to estimate the 3D helical configurations of the EAP actuator.
\end{abstract}

Index Terms-Electroactive polymer (EAP) actuators $\cdot$ soft and smart actuators - lamina-emergent mechanism - soft robotics $\cdot 3 \mathrm{D}$ kinematic modelling

\section{INTRODUCTION}

S PIRAL and helical geometries are two of the most common structures found in various biological systems. DNA and most of the protein structures have helical configurations [1]; bacteria (e.g. Escherichia coli) propels itself by forming its flagella filament into helical forms, sea shells form 3D spiral structures (e.g. Pearly Nautilus), rotating galaxies in astronomy (e.g. Fibonacci spiral), and climbing or twining plants grow spiral and helical tendrils [2]. The more a biologically inspired mechanism or device behaves like its natural counterpart, the higher is the complexity of the system; not only mimicking its kinematic behavior, but also its modelling becomes more challenging. Smart and preferably soft materials can be employed to establish actuators which can drive biologically inspired systems. Such soft and smart materials should have sufficient structural strength and internal (i.e., built-in)

R. Mutlu (rm991@uowmail.edu.au) is with School of Mechanical, Materials and Mechatronic Engineering, University of Wollongong, NSW, 2522, Australia

G. Alici (Corresponding Author) is with School of Mechanical, Materials and Mechatronic Engineering and ARC Centre of Excellence for Electromaterials Science, University of Wollongong, NSW, 2522, Australia (ph: +6142214115, gursel@uow.edu.au).

W.Li (weihuali@uow.edu.au) is with School of Mechanical, Materials and Mechatronic Engineering, University of Wollongong, NSW, 2522, Australia actuation and/or sensing capability with a low foot-print.

There is a growing research interest in soft robotics as an emerging field of robotics where a biological system or mechanism is mimicked by a robotic device or system with a soft structure and transducers. Soft robotic manipulator and actuator investigations in the literature have employed conventional means, in which working principles of an octopus arm [3, 4], an eel [5], an elephant trunk [6-9] or a snake $[10,11]$ are taken as a reference, to establish them. In addition to this, significant efforts have been devoted to modelling these biologically inspired soft robotic manipulators [12-19]. Most of these soft robotic manipulators in the literature have been built by serially connecting rigid parts driven by electric motors or cables (tendons), pneumatic actuators or a combination of them in order to generate biologically inspired motion of the soft manipulator. Recently, Tolley et al. constructed a pneumatically powered untethered soft robot by exploiting silicone elastomer material as an actuator [20].

A bioinspired robotic concept can be realized by strategically incorporating built-in actuation, sensing and control into a soft monolithic body. EAPs are soft smart materials with built-in actuation and have extraordinary properties including suitability to miniaturization [21-23]. They can easily adapt to unstructured environments (e.g. as an active catheter) due to their soft and highly flexible structure. An EAP actuator can be a good candidate for bioinspired micro robotic applications such as a bacteria type swimming robot [24] or a drug delivery system [23]. Though there are a few types of EAP actuators, bending type is the most common one in which the EAP actuator has two active polymer layers grown on both sides of a constraining passive porous polymeric layer containing the electrolyte needed for electrochemomechanical operation of the EAP actuator. Most of the studies in the literature focus on the cantilever beam like configuration of the bending type EAP actuator. Cantilever beam approach is reasonably less complicated to model the EAP actuator. When an electrical input is applied to the electroactive polymer layers, these layers change their volume and this results in a mechanical movement (bending) of the actuator. When the electroactive polymer actuator is designed in a suitable shape which is initially flat (planar) and actuated, it can generate conformal shapes such as bacteria flagella-like helical shapes. 


\section{A. Previous studies on modelling of the EAP actuators}

The EAP actuators exhibit many similarities to those biological systems, which are in the form of helices, in terms of working principles. In our previous work [24], we have demonstrated a polypyrrole (PPy) based EAP polymer actuator forming helical configurations from an initially planar spiral form as a first prototype. PPy is one of the most commonly used electroactive polymer to fabricate ionic type EAP actuators. Li et al. demonstrated an active stent articulated by an Ionic Polymer Metal Composite (IPMC) actuator fabricated as a bi-normal helix that the helical IPMC actuator exhibits a radial expansion [25]. Carpi et al. used a dielectric elastomer actuator which is cut into a normal helix shape from a dielectric elastomer tube in order to produce an axial contraction (i.e. -5\%) [26]. Tadesse et al. [27] proposed artificial facial muscles based on helical polymer actuators fabricated using a method proposed by Ding et al. [28].

Virtually all modelling studies reported in the literature are for the EAP actuators or smart actuators operating in 2D. These modelling studies can be classified into two groups: mechanical and electrical. For the mechanical modelling, Pei and Inganas [29] used Timoshenko's classical bending beam theory to estimate the bending displacement of the bi-layer polymer actuators. When the EAP actuators produce some strain (therefore bending), the radius of the curvature formed by the actuator is measured and used to calculate the magnitude of the strain. This approach was adopted by Benslimane et al. [30], Madden [31] and Alici et al. [32] for tri-layer EAP actuators. However, this approach is based on the assumptions of a small strain and constant modulus of elasticity. Alici also applied the classical beam theory taking non-linear effects into account to estimate the non-linear steady-state bending displacements of the PPyEAP actuators [33]. Alternatively, bending type EAP actuators can be modeled by adapting a soft robotic approach, in other words, the actuator can be represented by a kinematically analogous curve (so-called backbone curve). Such modelling approach can be utilized for a single PPyEAP actuator [34, 35] not only for planar actuation but also for the three dimensional displacement of the soft actuator in order to estimate its conformal shapes. As the soft robotic modelling approach uses a high number of degrees of freedom to determine the soft actuator's or manipulator's kinematic configurations, the dynamic behavior of the soft system becomes more sophisticated. Modelling a highly deformable soft system with a regular geometry such as a cantilever beam operating in $2 \mathrm{D}$ can be less challenging than a system made of an irregular geometry operating in 3D such as the PPyEAP actuator considered in this study which shifts its initial shape from 2D to 3D as soon as it is activated. The actuator forms itself into a conical helix shape when electrically stimulated. This actuation mechanism can also be used as a lamina emergent mechanism [36].

A lamina emergent mechanism, as a subfield of compliant mechanisms, refers to a mechanism which is fabricated in one plane (lamina) and motion occurs in third dimension. For example, a laminated mechanism is constructed in $x y$ plane and the mechanism emerges out of the plane into $z$ axis direction when it is operated (e.g. "pop-up books"). Primary difference between the helix forming PPyEAP actuator and a lamina emergent mechanism is that the PPyEAP actuator has built-in actuation capability which we call an active-lamina emergent mechanism. The helical configurations of the PPyEAP actuator are varied by a potential difference as low as $1.5 \mathrm{~V}$ or lower depending on size of the actuator designed. The smaller is the size of the actuator, the lower the electric power required to fully bend the soft actuator. Once the shape is formed, the electrical input can be turned off; the helix preserves its shape- - thanks to the operation principle of this class of electroactive polymer actuators

This paper concerns, as an extension of our previous study [24], (i) building a soft robotic structure made from the ionic bending type EAP actuator which turns into helical 3D configurations from its initial 2D configuration upon actuation, and (ii) developing a 3D kinematic model and solving the model to estimate the actuator's helical configurations under electrical inputs lower than $1.5 \mathrm{~V}$. We present a kinematic model for this helix forming PPyEAP actuator in cylindrical coordinates using so-called backbone curve approach. The proposed model has been experimentally validated for the PPyEAP actuators with different sizes. The tip positions of the PPyEAP actuators have been obtained using an image processing system to estimate the whole helical configuration of the PPyEAP actuator by solving the kinematic model. The contribution of this study is to establish an effective kinematic model for the PPyEAP actuator as an active lamina emergent mechanism, which operates in dry environments, as opposed to its predecessors, and verify the model experimentally.

The helix forming PPyEAP actuator can be used for miniand micro-robotic swimming devices which mimic bacteria type swimming (as an artificial flagellum) to be used for drug delivery within the human body as illustrated in Fig. 1. The fabrication of the artificial flagellum is straightforward since the PPyEAP actuator is initially in a 2D configuration, cut in a spiral shape. The initial spiral shape of the actuator is cut from a bulk sheet of the PPyEAP bulk sheet using a laser system. The synthesis details of the PPyEAP sheet are outlined in Section 2.

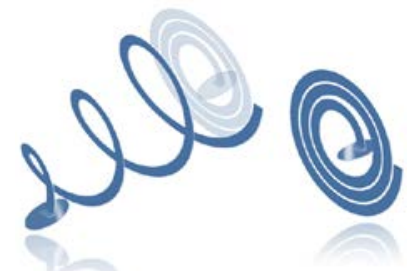

Fig. 1 Representation of a PPyEAP robotic bacterium (in its initial state in the right image and in its fully actuated state in the left image). 


\section{FABRICATION OF POLYMER ACTUATOR AND ITS WORKING PRINCIPLE}

Electroactive polymers have received significant research attention in recent decades due to their favorable chemical, electrochemical and mechanical properties to establish smart actuators. These smart materials are derived from monomers such as pyrrole, aniline or thiophene and others. These monomers have been used to establish various devices not only actuators but also sensors, membranes and energy storage structures [37-40].

A number of steps were followed to fabricate the electroactive polymer actuator considered in this study. First, the passive layer (i.e. polyvinylidene fluoride, PVDF) was sputter coated with a thin gold layer ( $\sim 100^{\circ} \mathrm{A}$ in thickness). PVDF is a non-conductive porous layer used for electrochemical cell separator and also holds the electrolytic ions (e.g., Li.TFSI) and is $110 \mu \mathrm{m}$ in thickness as received. In the meantime, a solution containing pyrrole monomer (typically $0.1 \mathrm{M}$ ), lithium triflouromethanesulfonimide $\left(\mathrm{Li}^{+} \mathrm{TFSI}^{-}, 0.1 \mathrm{M}\right.$ ) and $1 \%$ water in propylene carbonate (PC) was prepared for polymerization process. The gold coated PVDF was then submerged in this solution. The PPy layers were galvanostatically grown from the growth solution with a current density of $0.1 \mathrm{mAcm}^{-2}$ for 12 hours on the gold coated PVDF. This polymerization process generates a $30 \mu \mathrm{m}$ thick PPy layer on both sides of the gold coated PVDF. Hence, the total thickness of the PPy based electroactive polymer actuator is approximately $170 \mu \mathrm{m}$. The polymerization process is illustrated in Fig. 2. The designated spiral shapes are cut from the bulk sheet of the PPyEAP using a laser cutting system.

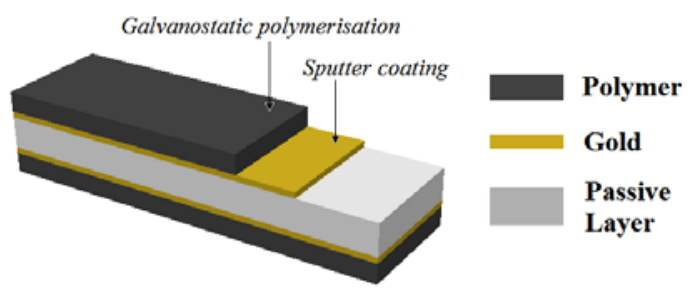

Fig. 2 Structure of the polypyrrole electroactive polymer (PPyEAP) actuator.

The operation principle of an ionic type PPy polymer actuator is based on converting electrical energy into the mechanical energy. When an electrical potential or current is applied to the PPy layers (working as electrodes) via the electrical contacts placed at one end of the PPy layers, a series of electro-chemo-mechanical reactions occur in the whole PPyEAP structure. This series of reactions cause the positively charged PPy layer to chemically oxidize and the negatively charged layer to chemically reduce (please refer to chemical reaction equation in Fig. 3). As the PPyEAP structure contains free ions $\left(\mathrm{TFSI}^{-}\right)$, $\mathrm{TFSI}^{-}$anions move from the electrolyte (mainly in the passive porous layer, please refer to Fig. 2) into the positively charged PPy layer and an opposite reaction occurs in the other PPy layer in order to electrically neutralize the PPy layers. As the $\mathrm{TFSI}^{-}$ anions rush into the positively charged PPy layer, this ion migration causes a volume expansion in the positively charged PPy layer and a volume contraction in the other PPy layer as TFSI $^{-}$anions move out of the negatively charged layer. These electro-chemo-mechanical reactions, therefore, generate a mechanical output (bending behavior due to the volume difference), as illustrated in Fig. 3.

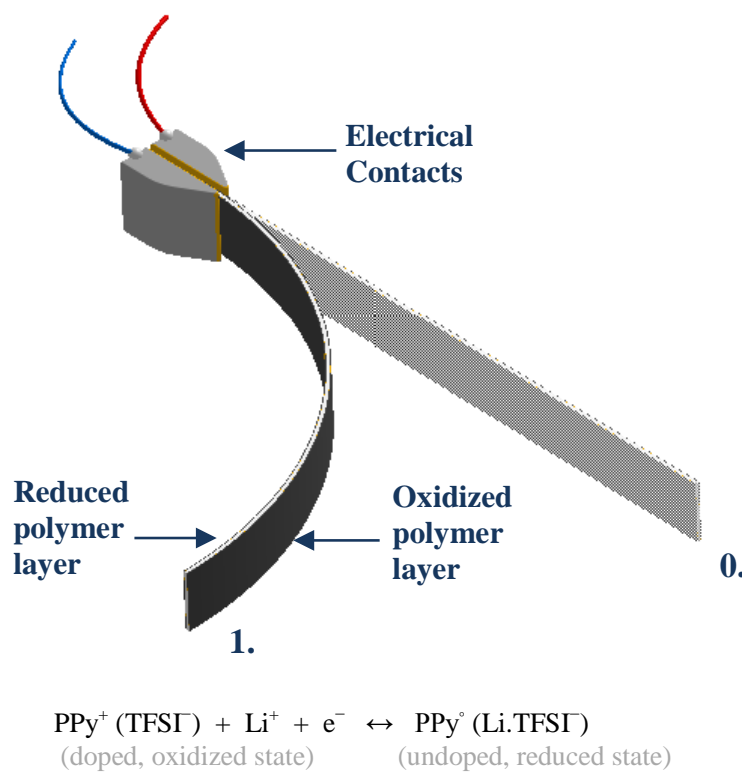

Fig. 3 Operation principle of a bending type PPyEAP actuator (0. Initial state and 1 . Maximum bent state).

\section{KinEMATIC ANALYSIS BASED ON BACKBONE CURVE APPROACH}

The actuators considered in this study generate highly nonlinear displacements when electrically stimulated. Kinematic representation of an EAP actuator, depending on its initial shape, can be like a hyper-redundant system (e.g. a tentacle if the EAP is used as a long strip) with a high degree of freedom (DoF). The higher is the degree of freedom, the higher is the computational power required to solve the kinematic model. However, every DoF does not need to be controlled in the EAP actuator's hyper redundant kinematic model. Although the EAP actuator can be actuated from several parts (i.e. multi input in order to obtain different mechanical output and shapes), most of the studies conducted on the EAP actuators activate the EAP actuator as a cantilever beam by fixing its one end and applying the electrical input at this fixed end. The helix forming PPyEAP actuator cut by a laser system are shown in Fig. 4. 


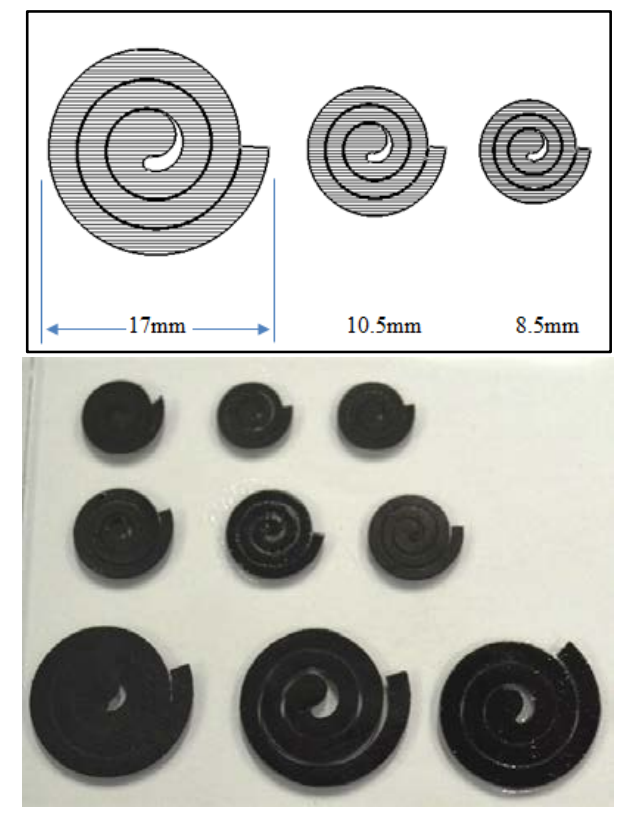

Fig. 4 Spiral PPyEAP actuators; sketches (top) and laser cut actuators (bottom) (upper row: $8.5 \mathrm{~mm}$, middle row: $10.5 \mathrm{~mm}$, lower row: $17 \mathrm{~mm}$ outer diameter).

Modelling and shape (i.e. topology) control of a soft and smart actuator turned into 3D active structure is problematic. Some shape control studies can be found in the literature with pre-designed shapes which restrict the shape of a soft robotic manipulator to certain conformations [10, 41, 42]. There is no previous study in the literature which reports on the kinematic modelling of a macro and micro-sized 3D soft and active actuator or manipulator based on the EAP actuators. Here, we derive a 3D kinematic model of the helix forming PPyEAP actuator analytically using differential geometry formulations combined with Denavit-Hartenberg transformations. This soft robotic kinematic model uses a backbone curve of the real soft PPyEAP actuator. We have derived the backbone curve in a discrete form for the purpose of the kinematic modelling. Following section describes the kinematics of a point (i.e. particle) moving along a spatial curve where orthogonal axes (i.e. local coordinate frame of the soft kinematic model) move with the point along the spatial curve. Next section integrates those local coordinate frames with the Denavit-Hartenberg convention to obtain the kinematic model.

\section{A. Kinematics of a point moving along a curve in $3 D$}

In classical differential geometry, a curve $\mathrm{C}(s, t)$ is parameterized by its arc length $\mathrm{s}[\theta(t)]$ in 3D Euclidean space in cylindrical coordinates [43]. Kinematics of a particle along the curve $\mathrm{C}(s, t)$ can be formulated from the tangent vector of the angular generalized coordinate $\theta(t)$ since the position vector of the particle is described by $\mathrm{R}(s, t)=\left[\mathrm{x}_{p}(s, t), \mathrm{y}_{p}(s, t), \mathrm{z}_{p}(s, t)\right]^{T}$. The tangent, normal and bi-normal unit vectors on the spatial curve $\mathrm{C}(s, t)$ are given by

$$
\text { Tangent; } \mathbf{t}=\mathrm{d} \mathbf{R} / \mathrm{ds}
$$

$$
\begin{aligned}
\text { Normal; } & \mathbf{n}=(\mathrm{d} \mathbf{t} / \mathrm{ds}) /\|(\mathrm{d} \mathbf{t} / \mathrm{ds})\| \\
\text { Bi-normal; } & \mathbf{b}=\mathbf{t} \times \mathbf{n}
\end{aligned}
$$

These unit vectors form the orthogonal coordinate axes (local coordinate frame) of a link along the spatial curve. As the point moves along the curve, the local coordinate frames are calculated using Equations (1-3). The higher is the number of positions of the point along the curve, the better is the kinematic representation. However, one should consider the amount of computation required when the number of links is excessive to approach the continuous form of the curve (Fig. 5). The Frenet-Serret formulation, which is given below, describes the motion of this local coordinate frame along the curve.

$$
\begin{aligned}
& \frac{\mathrm{d} \mathbf{t}}{\mathrm{ds}}=\kappa \mathbf{n}, \\
& \frac{\mathrm{d} \mathbf{n}}{\mathrm{ds}}=-\kappa \mathbf{n}+\tau \mathbf{b}, \\
& \frac{\mathrm{d} \mathbf{b}}{\mathrm{ds}}=-\tau \mathbf{b}
\end{aligned}
$$

where $\kappa$ is the curvature and $\tau$ is the torsion of the curve.

Once the local coordinate frames are determined from the parameterized equations of the curve, which are the elements of the position vector, the analytical model of the curve can be obtained using Denavit-Hartenberg transformations. A whole configuration of the soft robotic actuator/manipulator can then be determined using individual link configurations and the Denavit-Hartenberg transformations. The backbone curve of a typical helix forming soft actuator is illustrated in Fig. 5 in cylindrical coordinates including its local coordinate frames.

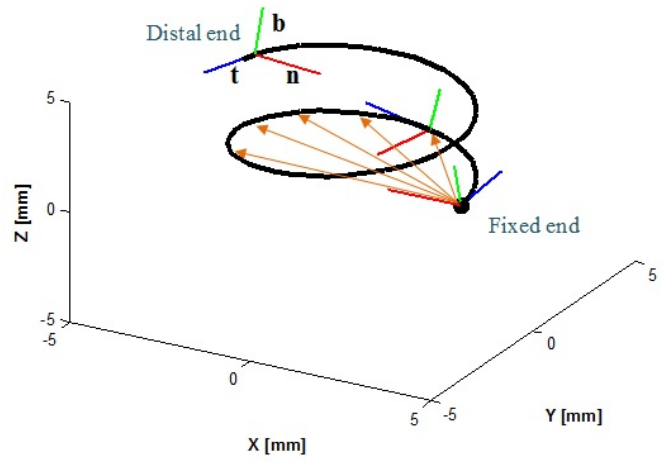

Fig. 5 Representation of the backbone curve of an actuator in the helical configuration with its local coordinate frames in cylindrical coordinates.

\section{B. Backbone Curve of PPyEAP Actuator in Cylindrical Coordinates}

The PPyEAP actuator has a rectangular cross-section with a constant width and thickness along the spiral (Fig. 6). If $r_{1}$ and $r_{2}$ are the starting and final radii of the conical helix, the position vector of each local coordinate system along the backbone curve of the conical helix can be described 
parametrically in the cylindrical coordinates as

$$
\mathrm{R}(s, t)=[\mathrm{x}(s, t), \mathrm{y}(s, t), \mathrm{z}(s, t)]^{T}
$$

where

$$
\begin{gathered}
x=\left(r_{1}+\frac{\gamma\left(r_{2}-r_{1}\right)}{2 l \pi}\right) \cos \gamma \\
y=q\left(r_{1}+\frac{\gamma\left(r_{2}-r_{1}\right)}{2 l \pi}\right) \sin \gamma \\
z=\frac{v \gamma}{2 l \pi}
\end{gathered}
$$

where $\gamma \in[0,2 \pi], l$ is the number of helix cycles, $q=+1$ for a right-handed helix and $v$ is the height of the helix [44]. In this study, we have used a left-handed spiral/helix in the kinematic models. The trihedron $(\boldsymbol{R} ; t, n, b)$ in the coordinate system $(\boldsymbol{O} ; x, y, z)$ is defined by Equations (1-3). The unit vectors are given by

$$
\begin{gathered}
\boldsymbol{t}=\left(a_{t}, b_{t}, c_{t}\right) \\
a_{t}=-\frac{\left(\left(r_{2}-r_{1}\right) \cos \gamma-\left(2 r_{1} l \pi+\gamma\left(r_{2}-r_{1}\right)\right) \sin \gamma\right)}{h} \\
b_{t}=-\frac{\left(q\left(r_{2}-r_{1}\right) \sin \gamma+\left(2 r_{1} l \pi+\gamma\left(r_{2}-r_{1}\right)\right) \cos \gamma\right)}{h} \\
c_{t}=-\frac{v}{h}
\end{gathered}
$$

where $h=\left(\left(r_{2}-r_{1}\right)^{2}+\left(2 r_{1} l \pi+\gamma\left(r_{2}-r_{1}\right)\right)^{2}+v^{2}\right)^{1 / 2}$

$$
\begin{gathered}
\boldsymbol{n}=\left(a_{n}, b_{n}, c_{n}\right)=\frac{\boldsymbol{t}_{\boldsymbol{r}} \times \boldsymbol{t}}{\left|\boldsymbol{t}_{\boldsymbol{r}} \times \boldsymbol{t}\right|} \\
\boldsymbol{b}=\left(a_{b}, b_{b}, c_{b}\right)=\frac{\boldsymbol{t} \times \boldsymbol{n}}{|\boldsymbol{t} \times \boldsymbol{n}|}
\end{gathered}
$$

where $\boldsymbol{t}_{\boldsymbol{r}}=\left(\left(r_{2}-r_{1}\right) \cos \gamma, q\left(r_{2}-r_{1}\right) \sin \gamma, v\right)$ is the direction vector of the surface of the conical helix formed by the actuator. The transformation matrices based on DenavitHartenberg representation are given by

$$
\begin{aligned}
& T_{x}(\varphi)=\left[\begin{array}{cccc}
1 & 0 & 0 & 0 \\
0 & \cos \varphi & q \sin \varphi & 0 \\
0 & -q \sin \varphi & \cos \varphi & 0 \\
0 & 0 & 0 & 1
\end{array}\right] \\
& T_{y}(\varphi)=\left[\begin{array}{cccc}
\cos \varphi & 0 & q \sin \varphi & 0 \\
0 & 1 & 0 & 0 \\
-q \sin \varphi & 0 & \cos \varphi & 0 \\
0 & 0 & 0 & 1
\end{array}\right]
\end{aligned}
$$

$$
T_{z}(\varphi)=\left[\begin{array}{cccc}
\cos \varphi & q \sin \varphi & 0 & 0 \\
-q \sin \varphi & \cos \varphi & 0 & 0 \\
0 & 0 & 1 & 0 \\
0 & 0 & 0 & 1
\end{array}\right]
$$

where $\varphi=\mathrm{k} \gamma \quad$ ( $k$ is the torsion factor). These transformation matrices represent the torsion about $t, n, b$ local coordinate axes of each link along the backbone curve. $T_{x}$ is used to obtain the torsion along the helical curve (lateral torsion). Then the position of a link, which is the center point of the cross section (Fig. 6), is calculated from $\boldsymbol{T}_{\boldsymbol{j}}(\varphi)$. The subscript $j$ indicates the torsion about the local coordinate axes. Also the position of a node on the surface of the model with respect to the base reference frame (fixed end of the PPyEAP actuator, Fig. 5) is calculated from

$$
\boldsymbol{p}(\alpha, \gamma)=\boldsymbol{r}(\alpha) \cdot \boldsymbol{T}_{\boldsymbol{j}}(\varphi) \cdot \boldsymbol{M}(\gamma)
$$

where $\boldsymbol{M}(\gamma)=\left[\begin{array}{llll}a_{t} & b_{t} & c_{t} & 0 \\ a_{b} & b_{b} & c_{b} & 0 \\ a_{n} & b_{n} & c_{n} & 0 \\ x_{R} & y_{R} & z_{R} & 1\end{array}\right]$, and $\boldsymbol{r}(\alpha)$ is the position vector of a node (with respect to its local coordinates) on a cross section along the backbone curve.

The rectangular cross section of the PPyEAP actuator is represented by 4 position vectors described in cylindrical coordinates, as shown in Fig. 6.

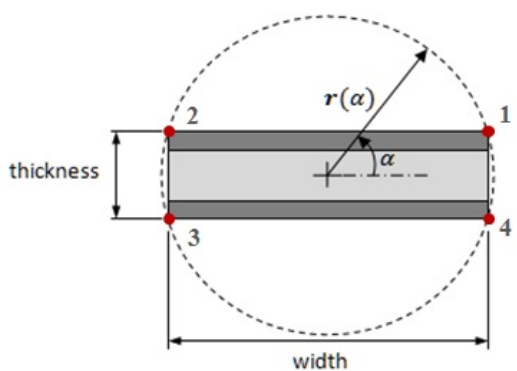

Fig. 6 Schematic of the position vector computation for a cross section of the PPyEAP actuator.

Equations (7-17) are used to estimate the bending configurations of the helix forming PPyEAP actuator. The whole kinematic representation of the initial shape of the actuator with its backbone curve is illustrated in Fig. 7 . Although a real PPyEAP actuator is a continuum system, it is represented as a hyper-redundant tentacle with a high number of links. As the model is developed in cylindrical coordinates, the number of links are determined by the number of helices (spiral coils) and more specifically by geometrical dimensions of the actuator's initial spiral shape. $r_{1}$ and $r_{2}$ (i.e. starting and final radii of the spiral, respectively) are the main parameters to determine the length of the actuator in the kinematic model. The width of the actuator determines the gap between the spiral coils. The number of spiral/helical coils is chosen to be 3 . The modelling approach requires the geometrical dimensions and number of spiral/helical coils first, and then dividing the resulting helices into a number of links making up the 
backbone curve. The longer is the overall length of the actuator, the higher is the number of links. The kinematic model should be solved for a few cases with different link lengths to ensure that the resulting discrete structure forms a smooth continuum structure, like a real actuator while the computation time is not excessive. In other words, the number of the links to construct the kinematic model of the actuator should be chosen to require a reasonably small computation time to solve the kinematic model for the shape estimation while the number of the links should be sufficient to represent the actuator with a smooth backbone curve. A few trials are enough to determine the optimal number of the links and their lengths.

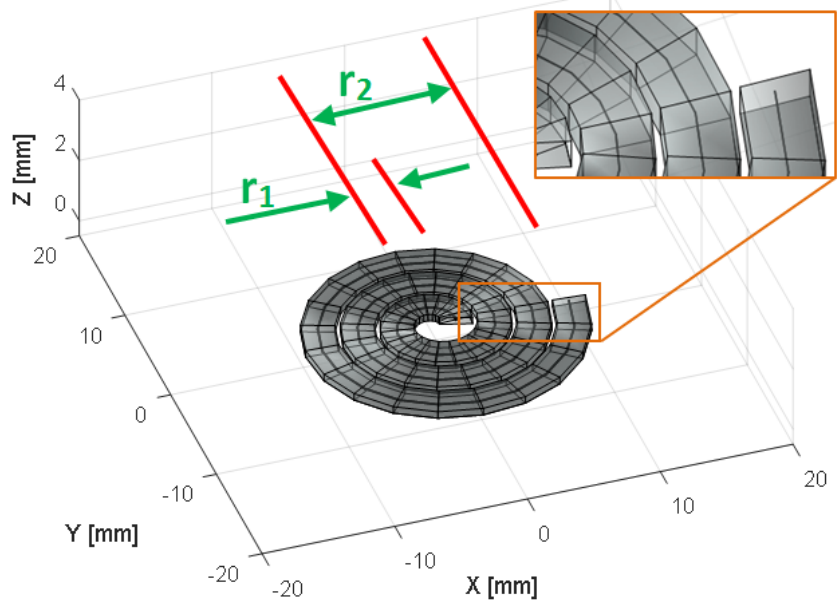

Fig. 7 Initial spiral shape of the actuator's kinematic model and its geometrical dimensions. The backbone curve is the thick line passing through the spiral in the zoom in drawing in the top right corner.

A typical helix formed by the PPyEAP actuator and its initial spiral configuration are illustrated in Fig. 8. Actuating the actuator only from its one end (in the center) dramatically reduces the hyper-redundancy of the kinematic model. However, another problem arises, which is the shape control of the PPyEAP actuator. It must be noted that EAP actuators are very flexible that their bending behavior highly depends on their physical properties. Thickness, width, length, and initial shape play a significant role in determining the final shape of the soft actuator. In order to estimate the helical configurations of the actuator in 3D, the kinematic model is solved for an inverse kinematic problem. The helical configuration of the actuator (in fact the backbone curve and its torsion along the backbone curve) is described as a discrete 3D curve; in other words, as a 60-link hyper-redundant manipulator like a kinematic chain. While there is no specific reason for choosing 60 links to construct the spiral and helical kinematic configurations of the actuator, this number is arbitrarily chosen to form a smooth representation of the 3-coil spiral/helix kinematic model of the actuator.

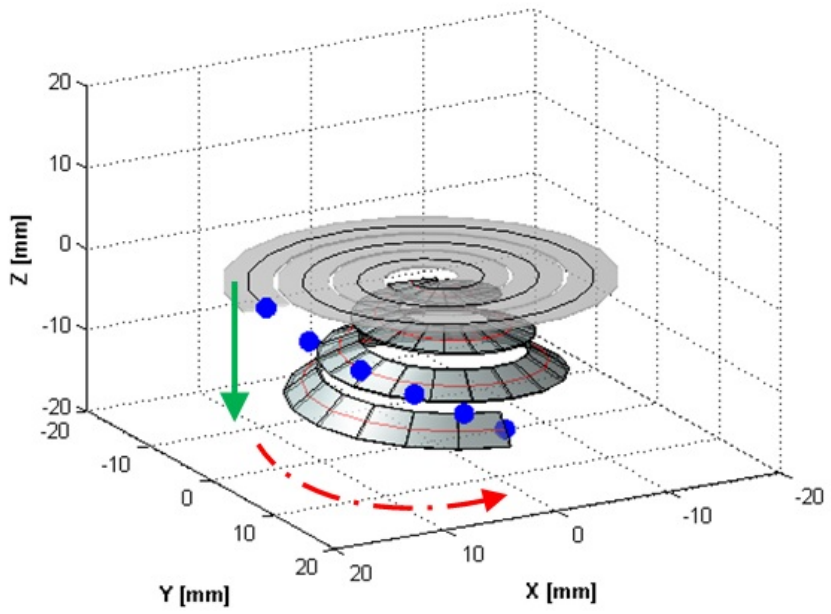

Fig. 8 Bending and twining behaviors of a typical right-handed PPyEAP actuator's kinematic representation with torsion effect.

By controlling the height parameter $v$ of the helix in the kinematic model, the final shape and the transient forms between the initial and final configurations are calculated using the kinematic model. It must be noted that the length of the actuator has a fixed torsion effect along its helical backbone which is taken into account within the model to estimate not only bending but also twisting behavior of the actuator. Bending rate which is the displacement along $z$ axis (Fig. 8) is calculated using Eq. 10 which depends on $v$ (height parameter determining displacement ratio with $\gamma$ ). The torsional effect which is proportional to the bending rate is calculated using Eq. 14 where the fixed torsion factor, $k$, directly rotates a local coordinate frame (therefore the cross section in that local coordinate frame) about the local tangential axis, $t$. The procedure followed to build the kinematic model of the helix forming PPyEAP actuator in cylindrical coordinates is depicted in an algorithm in Fig. 9.

\section{Parametric model}

The PPyEAP actuators have multi-domain propertieselectrical, chemical and mechanical. It would be ideal to incorporate all of these properties in a unified model describing their dynamic behavior under an electrical input. However, such a model will be quite complex to establish and computationally expensive to solve in order to understand the dynamic behavior of the actuators in the time and frequency domains. Here we propose a kinematic model only to determine the final 3D helical configurations of the actuator from its initially planar configuration as a function of time. We employ a parametric model to estimate the positions of the free end of the actuator at $r_{2}$ for a given input voltage. By incorporating the parametric model into the kinematic model, the helical configurations of the PPyEAP actuator can be estimated as a function of time. A quintic polynomial has been used for the parametric model which is expressed as 


$$
\left[\begin{array}{l}
\mathrm{X} \\
\mathrm{Y} \\
\mathrm{Z}
\end{array}\right]=\left[\begin{array}{l}
\mathrm{A}_{0} \mathrm{t}_{V}^{0}+\mathrm{A}_{1} \mathrm{t}_{V}^{1}+\mathrm{A}_{2} \mathrm{t}_{V}^{2}+\cdots+\mathrm{A}_{5} \mathrm{t}_{V}^{5} \\
\mathrm{~B}_{0} \mathrm{t}_{V}^{0}+\mathrm{B}_{1} \mathrm{t}_{V}^{1}+\mathrm{B}_{2} \mathrm{t}_{V}^{2}+\cdots+\mathrm{B}_{5} \mathrm{t}_{V}^{5} \\
\mathrm{C}_{0} \mathrm{t}_{V}^{0}+\mathrm{C}_{1} \mathrm{t}_{V}^{1}+\mathrm{C}_{2} \mathrm{t}_{V}^{2}+\cdots+\mathrm{C}_{5} \mathrm{t}_{V}^{5}
\end{array}\right]
$$

where $t$ is time, $A_{n}, B_{n}$ and $C_{n}$ are the input-voltage dependent coefficients, and $\mathrm{V}$ indicates the electrical input. The reason why we have chosen a quantic polynomial is that it is the minimum order polynomial to accurately represent the relationship between the electrical input and the Cartesian coordinates of the free end of the actuator.

\section{EXPERIMENTAL RESULTS AND DISCUSSION}

Experiments were conducted to evaluate the helix forming motion of the actuator. Experimental work was accomplished (i) to evaluate the feasibility of the actuator's ability to form a helix from its planar spiral shape and (ii) to quantify the efficacy of the kinematic model for the actuator with different spiral dimensions. The kinematic model uses 60 links to form the actuator's 3-coil spiral configuration smoothly. It should be noted that the kinematic model of the actuator is left-handed; $q=-1$ in Eq.9.

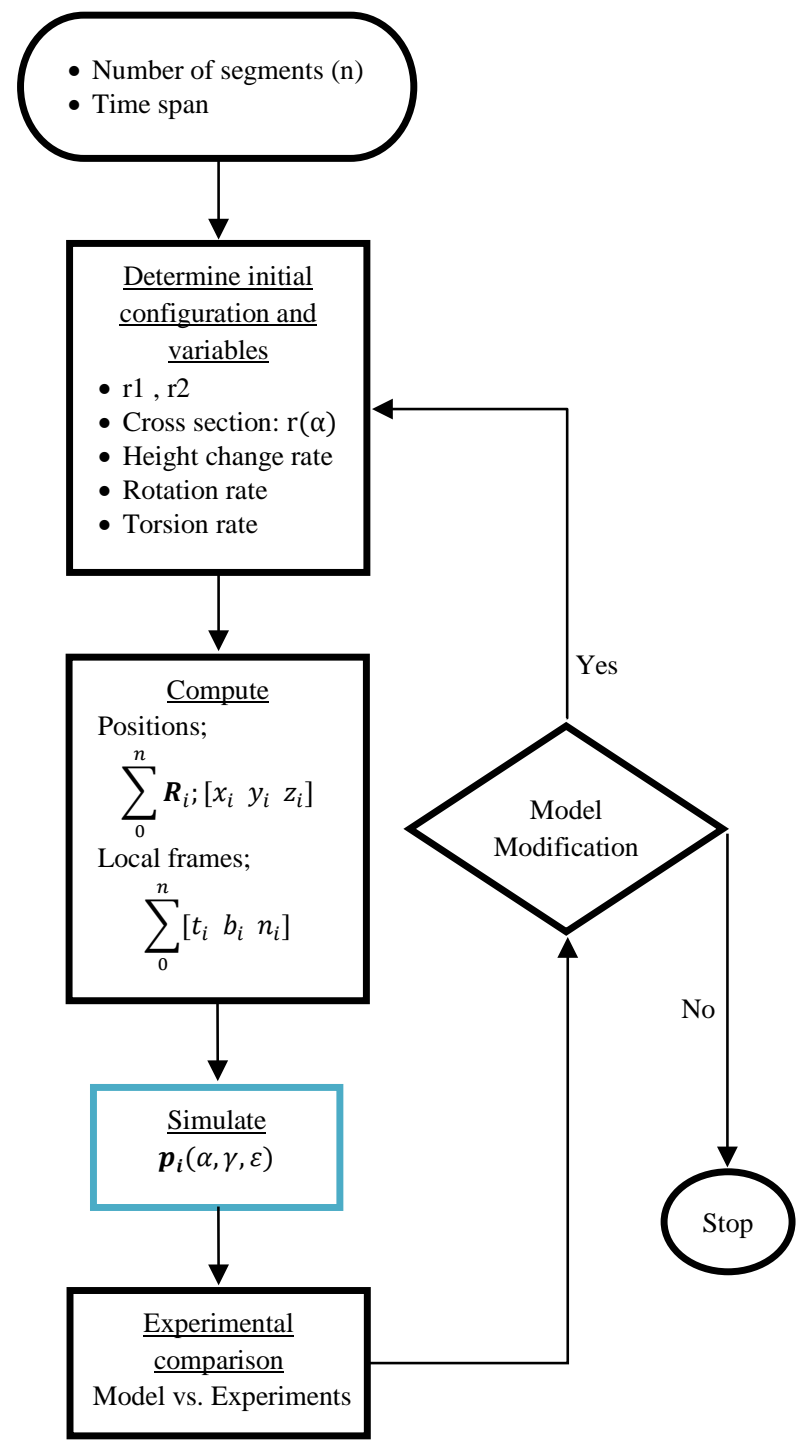

Fig. 9 Algorithm for the kinematic model development for the helix forming PPyEAP actuator.

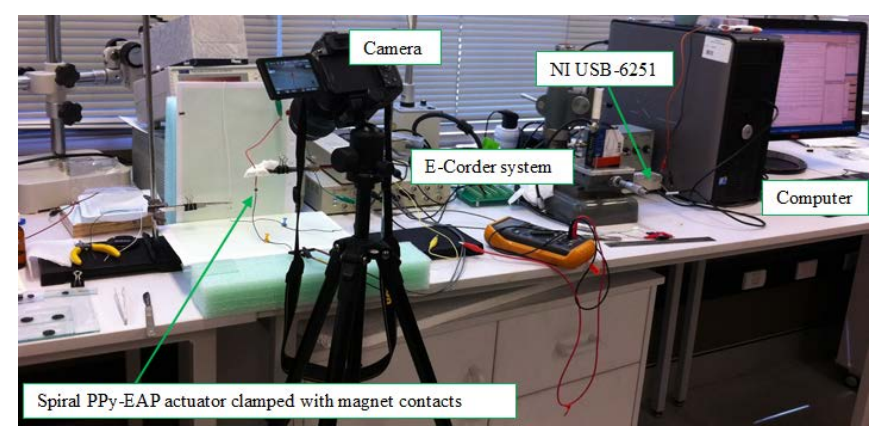

Fig. 10 Experimental setup for the helix forming PPyEAP actuator's actuation, and displacement measurement and analysis.

\section{A. Experimental setup and testing the helix forming PPyEAP}

A number of spiral actuators were cut from the PPyEAP bulk sheet using a laser cutting procedure. The experimental work for estimating the helical configurations of the actuator can be divided into four parts; (i) stimulating the actuator under a step voltage, (ii) recording the bending motion of the actuator with a digital camera aligned perpendicular to the bending motion plane of the actuator (i.e. bending and twining in the vertical direction) and analyzing the videos to extract the tip positions of the helix forming actuator, (iii) Incorporating the parametric model into the kinematic model, and (iv) solving the kinematic model to obtain a shape correspondence between the simulated helical configuration and the real helical configuration of the actuator and identify the kinematic model parameters for a satisfactory shape correspondence. The experimental setup for actuating the actuator is presented in Fig. 10. The electrical signals were generated in SIMULINK and applied through a potentiostat to the central part of the actuator to stimulate the actuator.

As the stimulated actuator forms 3-dimensional (i.e. helical) configurations, a multi camera system should ideally be used to obtain the coordinates of the tip of the actuator (e.g. 3 cameras can be placed for each axis, recording the $\mathrm{x}$, $\mathrm{y}, \mathrm{z}$ coordinates.). However, we used two cameras; first one to record the vertical displacements only and the second one to record the isometric view. While the vertical displacement videos were used in the image processing, the isometric view camera was used to obtain a more general picture of the whole helical configuration formed by the actuator. The images recorded by the first camera were analyzed to determine the vertical displacements and also the rotation of the actuator due to the torsion effect along the actuator length. The rotation about the $\mathrm{z}$-axis was obtained by analyzing the images recorded by two cameras.

Permanent magnet electrical contacts were used to clamp the spiral PPyEAP actuator prior to its stimulation. Two different contact pairs were used; (i) $1 \mathrm{~mm}$ in diameter and (ii) $3 \mathrm{~mm}$ in diameter which is gold coated, and they are shown in Fig. 11. It must be noted that, the contact-pair in the middle has a smaller contact area than the pair on the left 
in Fig. 11. $3 \mathrm{~mm}$ in diameter gold coated contact pair (in the middle in Fig. 11) has several advantages over the other two contact pairs. This contact pair clamps stronger than the contact pair $1 \mathrm{~mm}$ in diameter, therefore, more responsive is the actuator under an electrical stimulus. Also, this contact pair has a smaller contact resistance (due to gold coating) and is a better deterrent against the corrosion which increases the contact resistance significantly.

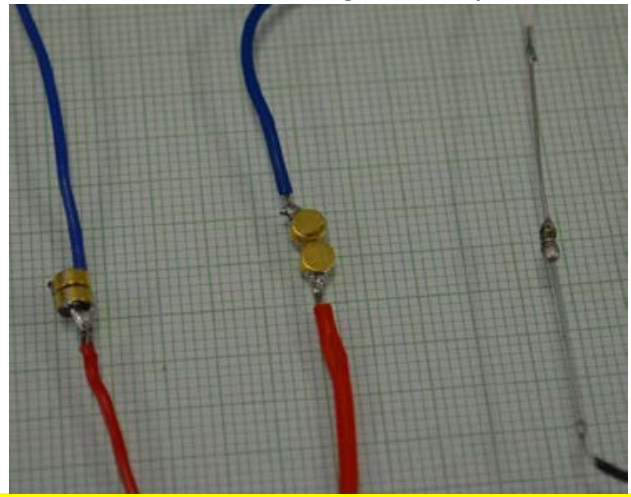

Fig. 11 Permanent magnet electrical contacts in different configurations and sizes.

\section{B. Experimental validation}

Prior to each test, the actuator was stimulated by a sine wave input voltage. The amplitude of the voltage input was increased incrementally from 0 to $1.5 \mathrm{~V}$. This procedure was performed after fabrication in order to enhance the bending capability of the actuator and reduce any hysteretic behavior [5]. A step voltage of $1.5 \mathrm{~V}$ was applied to stimulate the actuators for every test and their helix forming motion was captured with the camera.

The motion of the actuator was recorded as a video and image frames were extracted from each motion video. Although the camera could record at a rate of $30 \mathrm{fps}$, the image frames were recorded at a lower fps to simplify the image processing process in order to capture the motion of the actuator from its initial flat state to the final fully bent state. The tip positions of the actuator are extracted by analyzing the motion images. The parametric model is then obtained for the inverse kinematic calculation of all configurations of the actuator. The actuator's experimental and corresponding simulated configurations, which are estimated using the kinematic model, are presented in Figures 13 - 18 (for $17 \mathrm{~mm}, 10.5 \mathrm{~mm}$ and $8.5 \mathrm{~mm}$ outer diameter, respectively). Also, the sequential twining motion of the helix forming actuator with $17 \mathrm{~mm}$ outer diameter is given in Appendix A. A sequential motion of the helix forming actuator with an outer diameter of $17 \mathrm{~mm}$ is estimated using the parametric model obtained from the experimental measurements. The parametric model obtained for the $17 \mathrm{~mm}$ actuator is given in Eq.19. The experimental and the estimated tip positions of the $17 \mathrm{~mm}$ helix forming actuator are shown in Fig. 12. It must be noted that the kinematic model does not contain the electrical properties of the actuator. Nevertheless, Eq.19 which is a quintic polynomial model estimating the actuator tip positions as a function time for a given electrical input describes the relation between the electrical input and resulting output, which is the output of the kinematic model.

$$
\left[\begin{array}{l}
\mathrm{X} \\
\mathrm{Y} \\
\mathrm{Z}
\end{array}\right]=\left[\begin{array}{c}
0.0012 \mathrm{t}_{V}+0.0014 \mathrm{t}_{V}^{2}-0.2249 \mathrm{t}_{V}^{3}-0.0176 \mathrm{t}_{V}^{4}+9.1819 \mathrm{t}_{V}^{5} \\
-0.0007 t_{V}+0.0199 \mathrm{t}_{V}^{2}+0.0108 \mathrm{t}_{V}^{3}-2.0228 \mathrm{t}_{V}^{4}+0.5776 \mathrm{t}_{V}^{5} \\
-0.0051 \mathrm{t}_{V}^{3}-0.4866 \mathrm{t}_{V}^{4}-4 \mathrm{t}_{V}^{5}
\end{array}\right]
$$
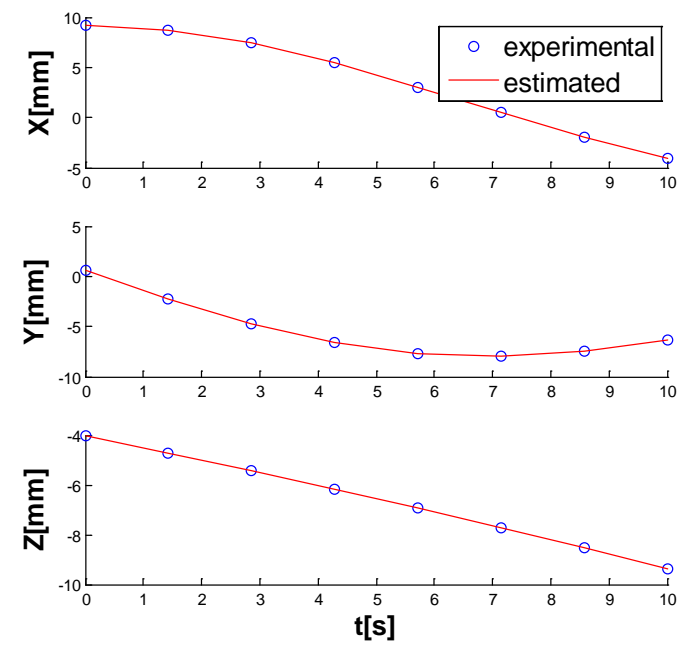

Fig. 12 Experimental and estimated tip positions of the helix forming PPyEAP actuator with 17mm outward radius by employing Eq.19.

It must be noted that the length of the spiral/helix is fixed in the model and the actuator configurations are calculated with the torsional effect along the helical curve using this fixed length constraint. It would be more desirable to compare the experimental and simulation results for the whole shape of the real actuator, not for its tip coordinates only. However, this would require a more sophisticated and expensive image processing system such as multiple cameras positioned along each axis as mentioned above and placing some indicative marks along the helical actuator in order to capture the helical motion more accurately and subsequently to conduct an error analysis between the real and estimated helical shapes of the actuator in order to quantify the efficacy of the kinematic model.

Experimental and simulation results provided in this study suggest that the backbone curve approach can be used to estimate the configurations of the EAP actuator not only in $2 \mathrm{D}$ but also in 3D. Its highly nonlinear displacement output can be estimated using the kinematic model proposed in this study.

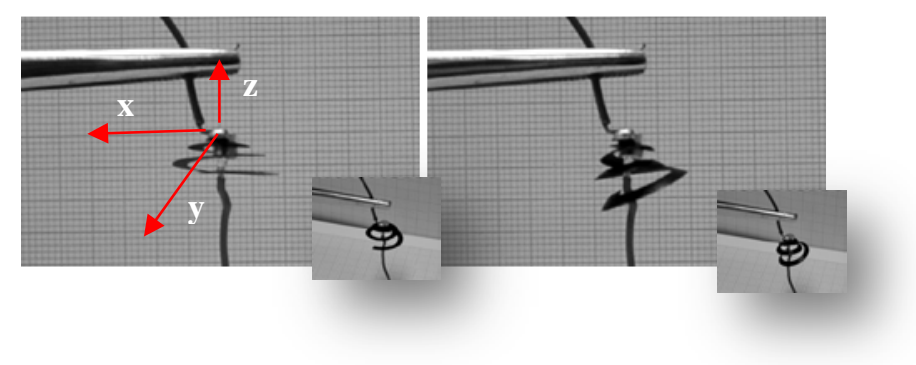


Fig. 13 Experimental results for the helix forming PPyEAP actuator's initial and final configuration (17 mm outer diameter). It must be noted that the actuator deflects under its own weight in its initial configuration.
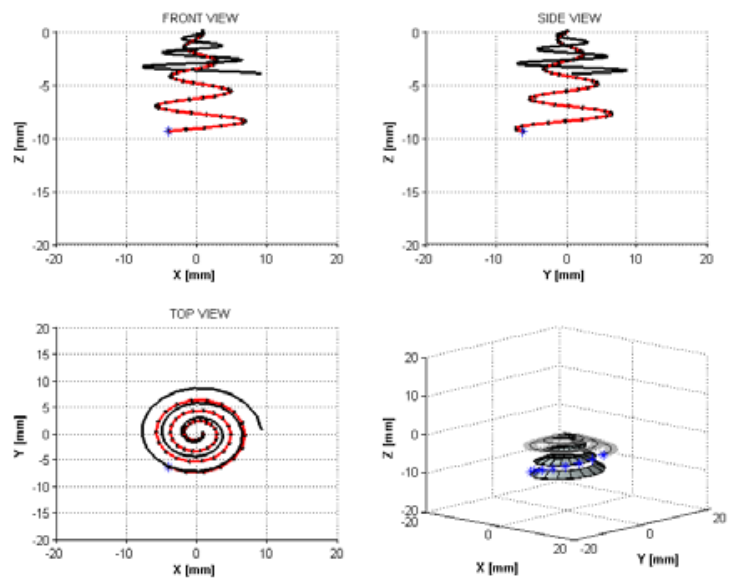

Fig. 14 Simulation results for the helix forming PPyEAP actuator (initial configuration, the black curve, and the final configuration, the red-dotted curve, of the actuator's backbone curve are shown in front and top views, $17 \mathrm{~mm}$ outer diameter. The blue star-marks are the experimentally measured tip positions).

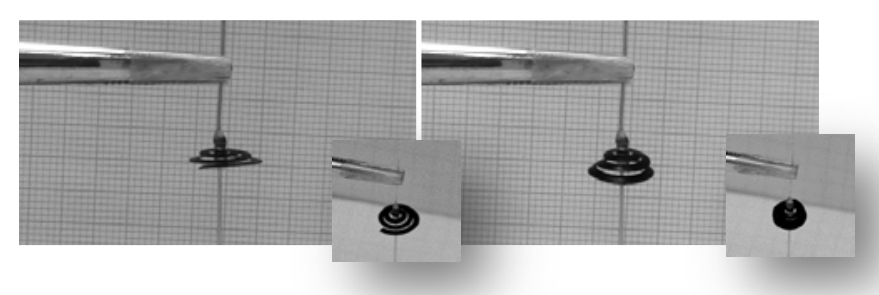

Fig. 15 Experimental results for the helix forming PPyEAP actuator's initial and final configurations (10.5 mm outer diameter).
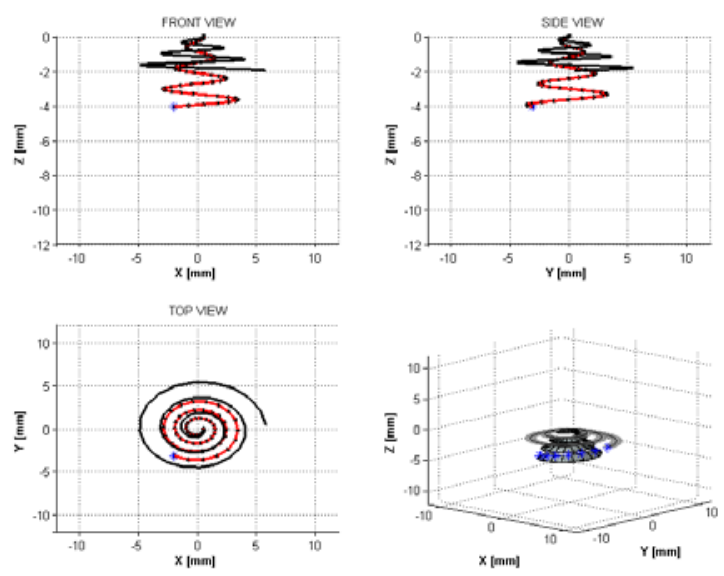

Fig. 16 Simulation results for the helix forming PPyEAP actuator (initial configuration, the black curve, and the final configuration, the red-dotted curve, of the actuator's backbone curve are shown in front and top views, $10.5 \mathrm{~mm}$ outer diameter. The blue star-marks are the experimentally measured tip positions).

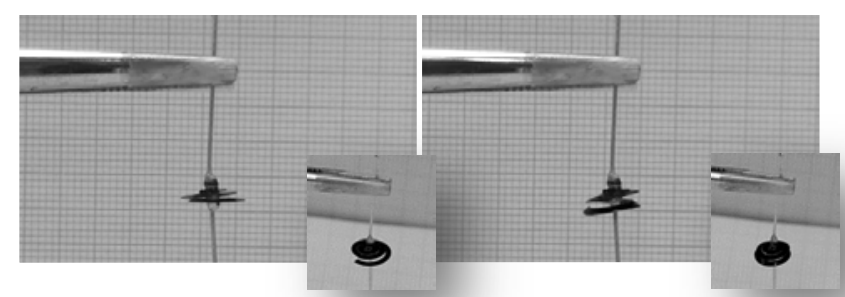

Fig. 17 Experimental results for the helix forming PPyEAP actuator's initial and final configurations (8.5 mm outer diameter).
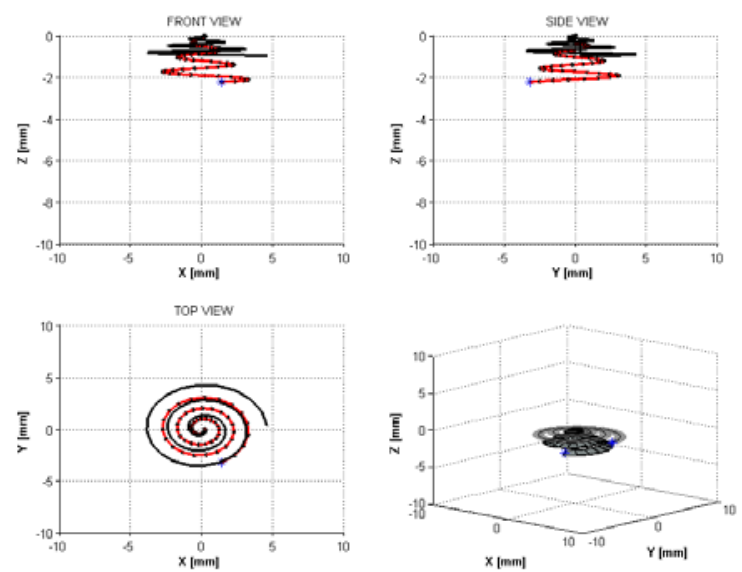

Fig. 18 Simulation results for the helix forming PPyEAP actuator (initia configuration, the black curve, and the final configuration, the red-dotted curve, of the actuator's backbone curve are shown in front and top views, $8.5 \mathrm{~mm}$ outer diameter. The blue star-marks are the experimentally measured tip positions).

\section{CONCLUSIONS AND FUTURE WORK}

This study shows that the bending type PPyEAP actuators can be fabricated such that they can change their configuration from a planar shape to a 3D shape in the form of a helix, upon stimulation. We have employed the backbone curve approach to establish a kinematic model for the helix forming motion of such actuators. The kinematic model combines the differential geometry with the conventional Denavit-Hartenberg transformations. Having the kinematic model in cylindrical coordinates reduces the number of independent variables in the model to the vertical displacement, rotation and torsion factors. Further, a parametric model is incorporated into the kinematic model. The kinematic model is solved to estimate the helical configurations of the actuator. Experimental results have demonstrated a satisfactory correlation with the simulation results. The initial configuration (i.e. no electrical input) of the actuators had some initial deflection due to their own weight; the smaller is the PPyEAP actuator spiral, the smaller is the initial deflection. As the actuator size is smaller, the final deflected (i.e. formed helix) shape has less deflection. The initial deflection of the actuator has also been considered in the kinematic model simulations and has been reflected in the results in Figures 13 - 18. This type of motion generated by artificial muscles is quite complex and challenging to model. However, the kinematic model proposed in this study is quite effective in predicting the 
helix forming behavior of these soft actuators.

Understanding the bending behavior of the EAP actuators forming 3D shapes from a 2D shape can open new applications such as micro swimmers with helical propellers, micro spring-suspension systems, micro mixers, motion converters, active-compliant lamina emergent mechanisms and combination of them. An example to an active lamina emergent mechanism articulated with the actuators considered in this paper can be an auto-focusing mechanism for a typical cell phone camera where a lens can be placed in the middle of one-piece PPyEAP soft mechanism [36]. This active-compliant mechanism can also be utilized as a micro linear positioner.

Future work includes miniaturizing the helix forming PPyEAP actuator in order to employ the helix forming topology within a soft robotic device such as a robotic swimmer or a motion converter. We also plan to develop a dynamic model of the helix forming actuator so that its realtime dynamic behavior can be understood and controlled accurately.

\section{ACKNOWLEDGEMENTS}

This work was supported in part by the Intelligent NanoTera Research Systems Laboratory and by ARC Centre of Excellence for Electromaterials Science (Grant No. CE0561616 and CE140100012).

\section{REFERENCES}

[1] J. D. Watson and F. H. C. Crick, "Molecular Structure of Nucleic Acids: A Structure for Deoxyribose Nucleic Acid," Nature, vol. 171, pp. 737-738, 1953.

[2] T. McMillen and A. Goriely, "Tendril Perversion in Intrinsically Curved Rods," Journal of Nonlinear Science, vol. 12, pp. 241-281, 2002.

[3] Y. Yekutieli, R. Sagiv-Zohar, R. Aharonov, Y. Engel, B. Hochner, and T. Flash, "Dynamic Model of the Octopus Arm. I. Biomechanics of the Octopus Reaching Movement," Journal of Neurophysiology, vol. 94, pp. 1443-1458, 2005.

[4] L. Margheri, C. Laschi, and B. Mazzolai, "Soft robotic arm inspired by the octopus: I. From biological functions to artificial requirements," Bioinspiration \& Biomimetics, vol. 7, p. 025004, 2012.

[5] F. Boyer, M. Porez, and W. Khalil, "Macro-continuous computed torque algorithm for a three-dimensional eellike robot," Robotics, IEEE Transactions on, vol. 22, pp. 763-775, 2006.

[6] M. W. Hannan and I. D. Walker, "Analysis and initial experiments for a novel elephant's trunk robot," in Intelligent Robots and Systems, 2000. (IROS 2000). Proceedings. 2000 IEEE/RSJ International Conference on, 2000, pp. 330-337 vol.1.

[7] M. W. Hannan and I. D. Walker, "Novel Kinematics for Continuum Robots," in Advances in Robot
Kinematics, J. Lenarčič and M. M. Stanišić, Eds., ed: Springer Netherlands, 2000, pp. 227-238.

[8] M. W. Hannan and I. D. Walker, "Kinematics and the Implementation of an Elephant's Trunk Manipulator and Other Continuum Style Robots," Journal of Robotic Systems, vol. 20, pp. 45-63, 2003.

[9] O. Salomon and A. Wolf, "Inclined Links HyperRedundant Elephant Trunk-Like Robot," Journal of Mechanisms and Robotics-Transactions of the Asme, vol. 4, Nov 2012.

[10] S. Hirose and M. Mori, "Biologically Inspired Snakelike Robots," in Robotics and Biomimetics, 2004. ROBIO 2004. IEEE International Conference on, 2004, pp. 1-7.

[11] B. Atakan, A. M. Erkmen, and I. Erkmen, 3-D grasping during serpentine motion with a snake-like robot, 2005.

[12] R. Kang, A. Kazakidi, E. Guglielmino, D. T. Branson, D. P. Tsakiris, J. A. Ekaterinaris, et al., "Dynamic model of a hyper-redundant, octopus-like manipulator for underwater applications," in Intelligent Robots and Systems (IROS), 2011 IEEE/RSJ International Conference on, 2011, pp. 4054-4059.

[13] Z. Tianjiang, D. T. Branson, E. Guglielmino, and D. G. Caldwell, "A 3D dynamic model for continuum robots inspired by an octopus arm," in Robotics and Automation (ICRA), 2011 IEEE International Conference on, 2011, pp. 3652-3657.

[14] T. Baba, Y. Kameyama, T. Kamegawa, and A. Gofuku, "A snake robot propelling inside of a pipe with helical rolling motion," in SICE Annual Conference 2010, Proceedings of, 2010, pp. 23192325.

[15] Z. Y. Bayraktaroglu, A. Kilicarslan, and A. Kuzucu, "Design and Control of Biologically Inspired Wheelless Snake-like Robot," in Biomedical Robotics and Biomechatronics, 2006. BioRob 2006. The First IEEE/RAS-EMBS International Conference on, 2006, pp. 1001-1006.

[16] G. Robinson and J. B. C. Davies, "Continuum robots a state of the art," in Robotics and Automation, 1999. Proceedings. 1999 IEEE International Conference on, 1999, pp. 2849-2854 vol.4.

[17] D. B. Camarillo, C. F. Milne, C. R. Carlson, M. R. Zinn, and J. K. Salisbury, "Mechanics Modeling of Tendon-Driven Continuum Manipulators," Robotics, IEEE Transactions on, vol. 24, pp. 1262-1273, 2008.

[18] Y. Sung-Weon and O. Il-Kwon, "A biomimetic jellyfish robot based on ionic polymer metal composite actuators," Smart Materials and Structures, vol. 18, p. 085002, 2009.

[19] J. C. Nawroth, H. Lee, A. W. Feinberg, C. M. Ripplinger, M. L. McCain, A. Grosberg, et al., "A tissue-engineered jellyfish with biomimetic propulsion," Nature Biotechnology, vol. 30, pp. 792797, Aug 2012.

[20] M. T. Tolley, R. F. Shepherd, B. Mosadegh, K. C. Galloway, M. Wehner, M. Karpelson, et al., "A 
Resilient, Untethered Soft Robot," Soft Robotics, vol. 1, pp. 213-223, 2014/09/01 2014.

[21] D. Trivedi, C. D. Rahn, W. M. Kier, and I. D. Walker, "Soft robotics: Biological inspiration, state of the art, and future research," Applied Bionics and Biomechanics, vol. 5, pp. 99-117, 2008/12/16 2008.

[22] B. Gaihre, G. Alici, G. M. Spinks, and J. M. Cairney, "Pushing the Limits for Microactuators Based on Electroactive Polymers," Microelectromechanical Systems, Journal of, vol. 21, pp. 574-585, 2012.

[23] E. W. H. Jager, E. Smela, and O. Inganäs, "Microfabricating Conjugated Polymer Actuators," Science, vol. 290, pp. 1540-1545, 2000.

[24] R. Mutlu, G. Alici, and L. Weihua, "Kinematic modeling for artificial flagellum of a robotic bacterium based on electroactive polymer actuators," in Advanced Intelligent Mechatronics (AIM), 2011 IEEE/ASME International Conference on, 2011, pp. 440-445.

[25] L. Song-Lin, K. Woo-Young, C. Tai-Hong, and O. IlKwon, "A helical ionic polymer-metal composite actuator for radius control of biomedical active stents," Smart Materials and Structures, vol. 20, p. 035008, 2011.

[26] F. Carpi, A. Migliore, G. Serra, and D. D. Rossi, "Helical dielectric elastomer actuators," Smart Materials and Structures, vol. 14, p. 1210, 2005.

[27] Y. Tadesse, R. W. Grange, and S. Priya, "Synthesis and cyclic force characterization of helical polypyrrole actuators for artificial facial muscles," Smart Materials and Structures, vol. 18, p. 085008, 2009.

[28] J. Ding, L. Liu, G. M. Spinks, D. Zhou, G. G. Wallace, and J. Gillespie, "High performance conducting polymer actuators utilising a tubular geometry and helical wire interconnects," Synthetic Metals, vol. 138, pp. 391-398, 2003.

[29] Q. Pei and O. Inganaes, "Electrochemical applications of the bending beam method. 1. Mass transport and volume changes in polypyrrole during redox," The Journal of Physical Chemistry, vol. 96, pp. 1050710514, 1992/12/01 1992.

[30] M. Benslimane, P. Gravesen, K. West, S. Skaarup, and P. Sommer-Larsen, "Performance of polymer-based actuators: the three-layer model," pp. 87-97, 1999.

[31] P. G. A. Madden, "Development and modeling of conducting polymer actuators and the fabrication of a conducting polymer based feedback loop," Ph.D. thesis, Dept. of Mechanical Engineering, Massachusetts Institute of Technology, 2003.

[32] G. Alici, B. Mui, and C. Cook, "Bending modeling and its experimental verification for conducting polymer actuators dedicated to manipulation applications," Sensors and Actuators A: Physical, vol. 126, pp. 396404, 2006.

[33] G. Alici, "An effective modelling approach to estimate nonlinear bending behaviour of cantilever type conducting polymer actuators," Sensors and Actuators B: Chemical, vol. 141, pp. 284-292, 2009.
[34] R. Mutlu, G. Alici, X. Xiang, and W. Li, "Electromechanical modelling and identification of electroactive polymer actuators as smart robotic manipulators," Mechatronics, vol. 24, pp. 241-251, 2014.

[35] R. Mutlu, G. Alici, and W. Li, "An effective methodology to solve inverse kinematics of electroactive polymer actuators modelled as active and soft robotic structures," Mechanism and Machine Theory, vol. 67, pp. 94-110, 2013.

[36] R. Mutlu, G. Alici, and W. Li, "A Soft Mechatronic Micro-Stage Mechanism Based on Electroactive Polymer Actuators," IEEE/ASME Transactions on Mechatronics, November 2015 (in print).

[37] G. Alici, G. Spinks, N. N. Huynh, L. Sarmadi, and R. Minato, "Establishment of a biomimetic device based on tri-layer polymer actuators--propulsion fins," Bioinspiration \& Biomimetics, vol. 2, pp. 18-30, 2007.

[38] Y. Wu, G. Alici, G. M. Spinks, and G. G. Wallace, "Fast trilayer polypyrrole bending actuators for high speed applications," Synthetic Metals, vol. 156, pp. 1017-1022, 2006.

[39] G. Alici, V. Devaud, P. Renaud, and G. Spinks, "Conducting polymer microactuators operating in air," Journal of Micromechanics and Microengineering, vol. 19, p. 025017, 2009.

[40] R. Mutlu and G. Alici, "A Multistable Linear Actuation Mechanism Based on Artificial Muscles," ASME Journal of Mechanical Design, vol. 132, p. 111001, 2010.

[41] G. S. Chirikjian and J. W. Burdick, "A modal approach to hyper-redundant manipulator kinematics," Robotics and Automation, IEEE Transactions on, vol. 10, pp. 343-354, 1994.

[42] S. R. Ahmadzadeh, "Modeling of hyper-redundant manipulators dynamics and design of fuzzy controller for the system," in Integration of Knowledge Intensive Multi-Agent Systems, 2005. International Conference on, 2005, pp. 248-253.

[43] E. Kreysig, "Theory of Curves," in Differential geometry, ed NY: Dover Publications, Incorporated, 1991, pp. 17-71.

[44] T. Olejnikova, "Cyclical surfaces created by a conical helix," Croatian Society for Geometry and Graphics, vol. 11, pp. 33-38, 2007. 
APPENDIX A

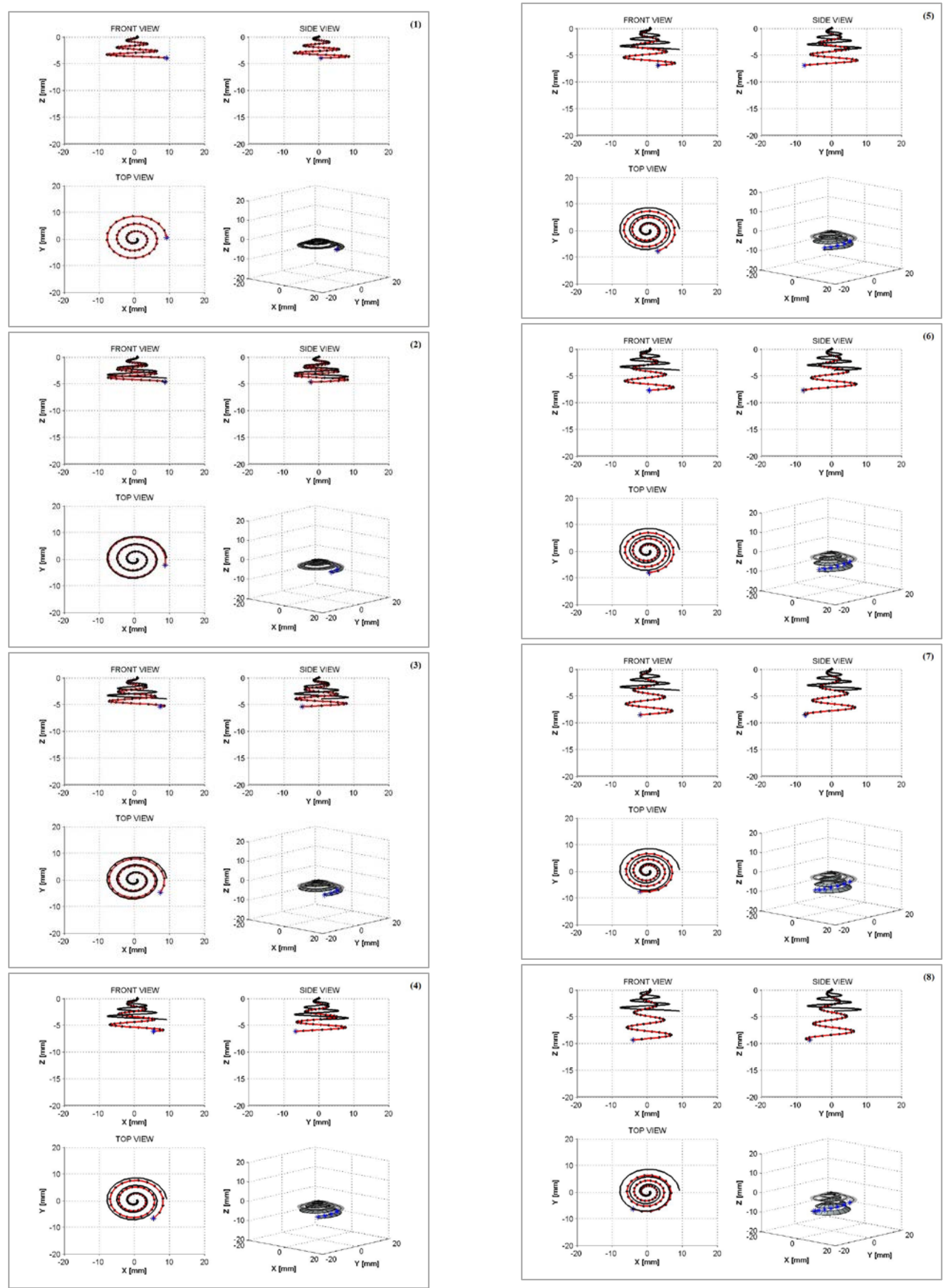

Sequential simulation results for the helix forming PPyEAP actuator, 17mm outer diameter (Blue star-marks are the experimentally measured tip positions). 